06

\title{
Синтез, кристаллическая структура и магнитные свойства соединения $\mathrm{YbFeTi}_{2} \mathrm{O}_{7}$
}

\author{
(C) Т.В. Дрокина ${ }^{1}$, Г.А. Петраковский ${ }^{1}$, М.С. Молокеев ${ }^{1,2,3}$, Д.А. Великанов ${ }^{1}$ \\ ${ }^{1}$ Институт фризики им. Л.В. Киренского ФИЦ КНЦ СО РАН, \\ Красноярск, Россия \\ ${ }^{2}$ Дальневосточный государственный университет путей сообщения, \\ Хабаровск, Россия \\ ${ }^{3}$ Сибирский фредеральный университет, \\ Красноярск, Россия \\ E-mail: tvd@iph.krasn.ru
}

(Поступила в Редакцию 28 июня 2017 г.)

\begin{abstract}
Описаны условия синтеза и результаты экспериментального исследования кристаллической структуры и магнитных свойств нового магнитного соединения $\mathrm{YbFeTi}_{2} \mathrm{O}_{7}$. Методом рентгеновской дифракции установлено, что кристаллическая структура исследуемого образца описывается ромбической пространственной группой Pcnb с параметрами элементарной ячейки: $a=9.8115(1) \AA ; b=13.5106(2) \AA ; c=7.31302(9) \AA$ и имеет место атомный беспорядок в распределении ионов железа $\mathrm{Fe}^{3+}$ по пяти структурным позициям. Результаты магнитных измерений в низкотемпературной области показывают излом на температурной зависимости магнитного момента и его зависимость от магнитной предыстории образца. Полученные экспериментальные данные позволяют предположить, что при понижении температуры происходит переход из парамагнитного состояния в спинстекольное магнитное состояние, характеризуемое температурой замерзания $T_{f}=4.5 \mathrm{~K}$ в образце с преимущественно антиферромагнитным обменным взаимодействием в спиновой системе. Варьирование химического давления при замещении редкоземельного иона $R \rightarrow \mathrm{Yb}$ в системе $R \mathrm{FeTi}_{2} \mathrm{O}_{7}$ не изменяет симметрию кристаллической решетки и магнитное состояние.
\end{abstract}

DOI: 10.21883 FTT.2018.03.45557.209

\section{1. Введение}

В связи с неослабевающим интересом к поиску новых материалов, перспективных для использования в различных областях техники и химической промышленности, синтез веществ с разнообразными магнитными свойствами и типами магнитного упорядочения остается одной из актуальных задач физики конденсированного состояния. В этом аспекте представляет интерес разработка и создание магнитных материалов, в том числе оксидных соединений, содержащих редкоземельные магнитные ионы и ионы переходных металлов и характеризуемых конкуренцией магнитных взаимодействий.

Известно, что новые магнитные соединения с общей формулой $R \mathrm{FeTi}_{2} \mathrm{O}_{7}$ (редкоземельный ион $R^{3+}=\mathrm{Sm}$, $\mathrm{Gd}, \mathrm{Dy}, \mathrm{Tu}, \mathrm{Tb}, \mathrm{Lu})$, кристаллическая симметрия которых описывается ромбической пространственной группой Pcnb, характеризуются атомным беспорядком в распределении магнитных ионов железа по различным структурным позициям и обладают магнитным состоянием спинового стекла [1-5]. Представляет интерес варьирование химического состава в системе $R \mathrm{FeTi}_{2} \mathrm{O}_{7}$, структурная и магнитная характеризация образцов с катионным замещением, приводящим к изменению химического давления.

В настоящей работе авторы приводят результаты синтеза и изучения свойств нового магнитного материала $\mathrm{YbFeTi}_{2} \mathrm{O}_{7}$. Представлены результаты рентгеновских и магнитных измерений.

\section{2. Синтез образцов и техника эксперимента}

Соединение $\mathrm{YbFeTi}_{2} \mathrm{O}_{7}$ получено реакцией в твердой фазе из смеси окислов $\mathrm{Fe}_{2} \mathrm{O}_{3}, \mathrm{Yb}_{2} \mathrm{O}_{3}$ и $\mathrm{TiO}_{2}$ при следующем соотношении, масс.\%: $\mathrm{Fe}_{2} \mathrm{O}_{3}-25.2$; $\mathrm{TiO}_{2}-12.6 ; \mathrm{Yb}_{2} \mathrm{O}_{3}-62.2$. Образцы в виде таблеток диаметром $10 \mathrm{~mm}$ и толщиной $1.5-2.0 \mathrm{~mm}$, подвергались высокотемпературной обработке при температурах $1200-1250^{\circ} \mathrm{C}$ и нормальном давлении. Процедура синтеза включает четыре отжига с промежуточным мокрым помолом в спиртовой среде и повторными этапами прессования. Химический и фазовый состав полученных образцов контролировался методом рентгеноструктурного анализа.

Порошковая рентгенограмма $\mathrm{YbFeTi}_{2} \mathrm{O}_{7}$ получена на дифрактометре D8 ADVANCE фирмы Bruker с использованием линейного детектора VANTEC и излучения линии $\mathrm{Cu} K_{\alpha}$. В эксперименте использовалась методика переменной скорости сканирования (VCT) и переменного шага (VSS). Время экспозиции увеличивалось с увеличением угла $2 \theta$, приводя к значительному улучшению качества отснятой рентгенограммы [6-8]. Как правило, на полуширине пика (FWHM) должно укладываться 5-8 экспериментальных точек. Однако пики значительно уширяются с увеличением угла $2 \theta$. Поэтому шаг был увеличен в высокоугловых областях $2 \theta$ с целью уменьшения затрат времени эксперимента [9]. Далее экспериментальные данные были конвертированы в один 
общепринятый в рентгенографии $X Y E$ файл, содержащий координаты $2 \theta_{i}$, интенсивность $I_{i}$ и стандартное отклонение $\sigma\left(I_{i}\right)$ для каждой экспериментальной точки. Уточнение Ритвельда, реализованное, например в программе TOPAS 4.2 [10], учитывает стандартное отклонение каждой точки посредством расчета веса для каждой точки $w_{i}=1 / \sigma\left(I_{i}\right)^{2}$. Таким образом, увеличение времени экспозиции для точки приводит к уменьшению стандартного отклонения $\sigma\left(I_{i}\right)$, и как следствие, к большему ее весу $w_{i}$ в уточнении методом наименьших квадратов. В методике VCT происходит выравнивание между собой весов слабых высокоугловых и сильных низкоугловых рефлексов, тогда как в обыкновенном эксперименте веса неравноценны и информация о структуре, содержащаяся в высокоугловой области теряется.

Экспериментальная рентгенограмма исследуемого образца $\mathrm{YbFeTi}_{2} \mathrm{O}_{7}$ была отснята по методике VCT/VSS и разбита на четыре части: $5-38.7^{\circ}$ (экспозиция в точке $3 \mathrm{~s}$, шаг $0.016^{\circ}$ ); $38.7-61.6^{\circ} \mathrm{C}$ (экспозиция в точке $9 \mathrm{~s}$, шаг $0.024^{\circ}$ ); 61.6-97.5 ${ }^{\circ}$ (экспозиция в точке $15 \mathrm{~s}$, шаг $0.032^{\circ}$ ); $97.5-140^{\circ}$ (экспозиция в точке $24 \mathrm{~s}$, шаг $0.040^{\circ}$ ). Общее время эксперимента составило $16 \mathrm{~h}$. Разбиение эксперимента на части было проведено в программе XRD Wizard [9]. Положение пиков определялось в программе EVA (2004 release) из программного пакета DIFFRAC-PLUS, поставляемого Bruker.

Эксперименты по определению температурного поведения магнитного момента выполнены на магнитометре MPMS-XL Сибирского федерального университета в интервале температур 2-300 K в магнитном поле 500 Ое.

\section{3. Экспериментальные результаты}

Структурные свойства поликристаллического соединения $\mathrm{YbFeTi}_{2} \mathrm{O}_{7}$ изучены методом рентгеновской дифракции, рентгенограмма показана на рис. 1. Данные

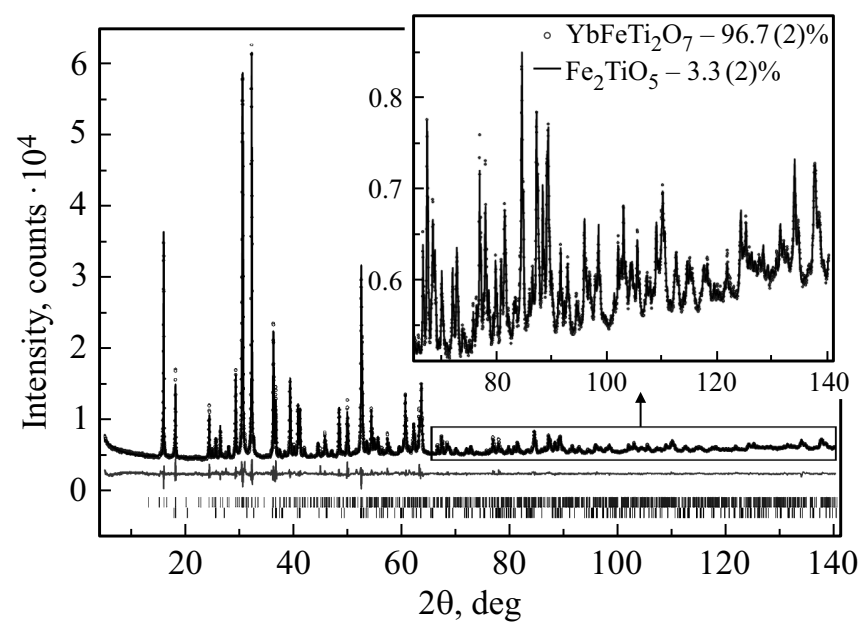

Рис. 1. Рентгенограмма поликристаллического соединения $\mathrm{YbFeTi}_{2} \mathrm{O}_{7}$ при комнатной температуре. Разностная рентгенограмма - нижняя кривая. Исследуемое вещество содержит $5.87 \%$ примеси состава $\mathrm{Fe}_{2} \mathrm{TiO}_{5}$.

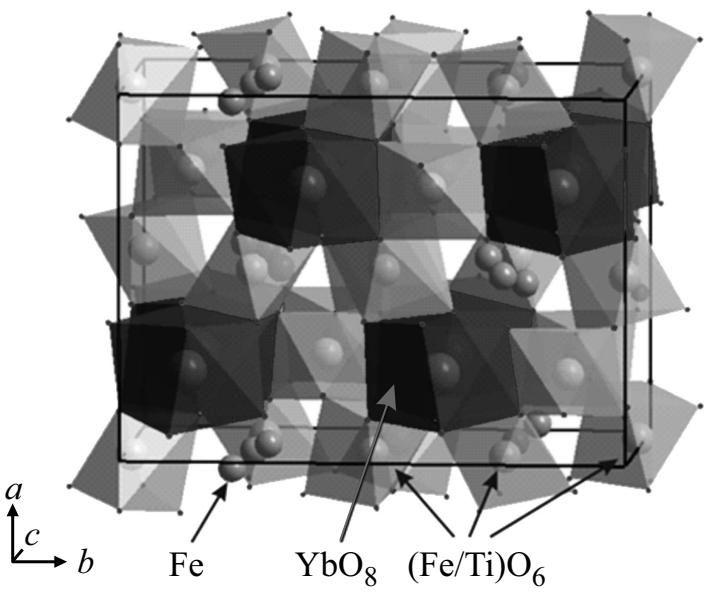

Рис. 2. Кристаллическая структура соединения $\mathrm{YbFeTi}_{2} \mathrm{O}_{7}$.

рентгеновского исследования синтезированного образца свидетельствуют о том, что кроме основной фазы $\mathrm{YbFeTi}_{2} \mathrm{O}_{7}$ исследуемый образец содержит в небольшом количестве $(3.3(2) \%)$ примесь состава $\mathrm{Fe}_{2} \mathrm{TiO}_{5}$.

Так как исследуемое соединение изоструктурно $\mathrm{GdGaTi}_{2} \mathrm{O}_{7}[11]$, то в качестве начальной модели кристаллической структуры материала $\mathrm{YbFeTi}_{2} \mathrm{O}_{7}$ была взята структура соединения $\mathrm{GdGaTi}_{2} \mathrm{O}_{7}$. Согласно данным рентгеновского исследования, кристаллическая симметрия образца $\mathrm{YbFeTi}_{2} \mathrm{O}_{7}$ при комнатной температуре описывается ромбической пространственной группой Pcnb. Следует отметить, что ион $\mathrm{Yb}^{3+}$ обладает наименьшим ионным радиусом $(R=1.00 \AA[12])$ среди магнитных редкоземельных ионов. Сравнение с другими известными соединениями системы $R \mathrm{FeTi}_{2} \mathrm{O}_{7}$ [1-5] показывает, что изменение химического давления, вызванного катионным замещением $R \rightarrow \mathrm{Yb}$, а также $\mathrm{Sm}, \mathrm{Gd}, \mathrm{Dy}, \mathrm{Tu}, \mathrm{Tb}$, $\mathrm{Lu}$, не изменяет симметрию кристаллической решетки цирконолитов.

Таблица 1. Основные кристаллографические характеристики $\mathrm{YbFeTi}_{2} \mathrm{O}_{7}$ и параметры рентгеновского эксперимента

\begin{tabular}{c|c}
\hline Пространственная группа & $P c n b$ \\
\hline$a, \AA$ & $9.8115(1)$ \\
$b, \AA$ & $13.5106(2)$ \\
$c, \AA$ & $7.31302(9)$ \\
$V, \AA^{3}$ & $969.41(2)$ \\
$Z$ & 8 \\
$D_{x}, \mathrm{~g}_{\mathrm{cm}^{3}}$ & 5.963 \\
$\mu, \mathrm{mm}^{-1}$ & 84.016 \\
$2 \theta$-интервал, & $5-140$ \\
Число рефлексов & 927 \\
Число уточняемых параметров & 74 \\
$R_{w p}, \%$ & 1.911 \\
$R_{\text {eхр }} \%$ & 0.671 \\
$R_{p}, \%$ & 1.751 \\
$G O F\left(\chi^{2}\right)$ & 2.850
\end{tabular}


Таблица 2. Координаты атомов, заселенности позиций $p$ и тепловые параметры $B_{\text {iso }}$ в соединении $\mathrm{YbFeTi}_{2} \mathrm{O}_{7}$

\begin{tabular}{c|c|l|l|l|l|l}
\hline Атом & $\begin{array}{c}\text { Кратность } \\
\text { позиции }\end{array}$ & \multicolumn{1}{|c|}{$x$} & $y$ & $z$ & $p$ & $B_{\text {iso, }} \AA^{2}$ \\
\hline Yb & 8 & $0.2459(3)$ & $0.13233(12)$ & $0.0040(5)$ & 1 & $0.57(8)$ \\
Ti1 & 8 & $0.2454(11)$ & $0.3847(4)$ & $0.4867(12)$ & $0.94(2)$ & $0.5(1)$ \\
Fe1 & 8 & $0.2454(11)$ & $0.3847(4)$ & $0.4867(12)$ & $0.06(2)$ & $0.5(1)$ \\
Ti2 & 4 & 0.5 & 0.25 & $0.248(3)$ & $0.59(5)$ & $0.5(2)$ \\
Fe2 & 4 & 0.5 & 0.25 & $0.248(3)$ & $0.41(5)$ & $0.5(2)$ \\
Ti3 & 8 & $0.0060(8)$ & $0.4858(4)$ & $0.2548(17)$ & $0.77(3)$ & $0.9(2)$ \\
Fe3 & 8 & $0.0060(8)$ & $0.4858(4)$ & $0.2548(17)$ & $0.23(3)$ & $0.9(2)$ \\
Fe & 4 & 0 & 0.25 & $0.327(2)$ & 0.78 & $0.7(2)$ \\
Fei & 8 & $0.025(6)$ & $0.278(4)$ & $0.175(9)$ & 0.11 & $0.7(2)$ \\
O1 & 8 & $0.1624(9)$ & $0.3909(11)$ & $0.248(6)$ & 1 & $1.0(2)$ \\
O2 & 8 & $0.4027(19)$ & $0.1112(15)$ & $0.252(6)$ & 1 & $1.0(2)$ \\
O3 & 8 & $0.109(2)$ & $0.1509(10)$ & $0.245(5)$ & 1 & $1.0(2)$ \\
O4 & 8 & $0.366(4)$ & $0.277(3)$ & $0.449(5)$ & 1 & $1.0(2)$ \\
O5 & 8 & $0.370(4)$ & $0.280(3)$ & $0.060(5)$ & 1 & $1.0(2)$ \\
O6 & 8 & $0.374(3)$ & $0.494(2)$ & $0.427(4)$ & 1 & $1.0(2)$ \\
O7 & 8 & $0.381(3)$ & $0.483(2)$ & $0.050(4)$ & 1 & $1.0(2)$
\end{tabular}

Основные кристаллографические характеристики и параметры эксперимента для соединения $\mathrm{YbFeTi}_{2} \mathrm{O}_{7}$ представлены в табл. 1. Координаты атомов и результаты уточнения заселенностей позиций атомов $p$ в материале $\mathrm{YbFeTi}_{2} \mathrm{O}_{7}$ содержатся в табл. 2. На рис. 2 приведена кристаллическая структура соединения $\mathrm{YbFeTi}_{2} \mathrm{O}_{7}$. Из табл. 2 следует, что в кристаллической структуре $\mathrm{YbFeTi}_{2} \mathrm{O}_{7}$ имеется пять неэквивалентных позиций атомов железа, причем три из них являются смешанными (Fe/Ti). Это способствует их хаотическому заселению ионами $\mathrm{Fe}^{3+}$.

Всего в элементарной кристаллической ячейке исследуемого соединения содержится $0.94 \times 8+0.59 \times 4$ $+0.77 \times 8=16$ атомов титана Ті и $0.06 \times 8+0.41 \times 4$ $+0.23 \times 8+(0.78 \times 4+0.11 \times 8)=8$ атомов железа Fе.

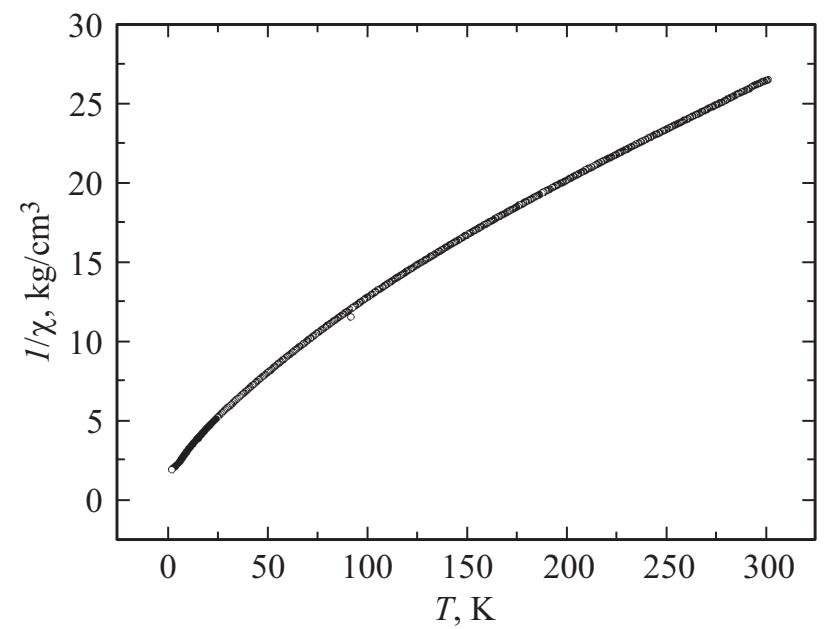

Рис. 3. Температурная зависимость обратной магнитной восприимчивости образца $\mathrm{YbFeTi}_{2} \mathrm{O}_{7}$, охлажденного в поле $H=0.05 \mathrm{~T}$. Масса образца $m=0.045 \mathrm{~g}$. Асимптотическая температура Нееля $\theta=-127 \mathrm{~K}$.

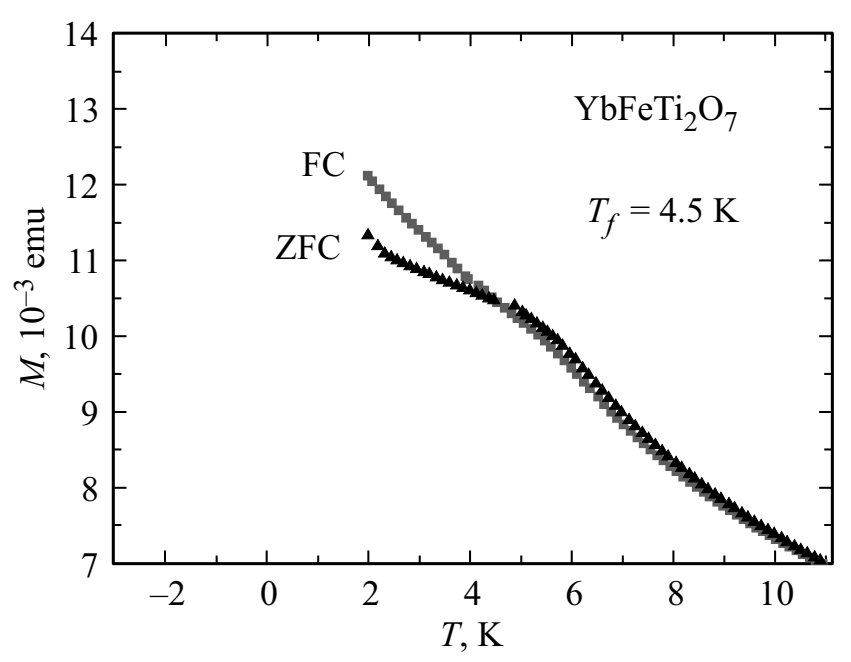

Рис. 4. Низкотемпературная зависимость магнитного момента $\mathrm{YbFeTi}_{2} \mathrm{O}_{7}$, полученная в двух режимах измерения: $\mathrm{FC}-$ охлаждение образца в магнитном поле $H=0.05 \mathrm{~T}$ и ZFC охлаждение без поля $(H=0)$. Измерения проведены в магнитном поле $H=0.05 \mathrm{~T}$. Масса образца $m=0.045 \mathrm{~g}$. Температура замерзания $T_{f}=4.5 \mathrm{~K}$.

Таким образом, учитывая относительные заселенности индивидуальных позиций, суммарную формулу можно записать в виде $\mathrm{YbFe}_{1.00(5)} \mathrm{Ti}_{2.00(5)} \mathrm{O}_{7}$.

Ионы титана в многоатомном кристалле $\mathrm{YbFeTi}_{2} \mathrm{O}_{7}$ находятся в диамагнитном состоянии $\mathrm{Ti}^{4+}$. Магнитная подсистема изучаемого соединения формируется ионами двух типов: редкоземельными ионами иттербия $\mathrm{Yb}^{3+}$ (электронная конфигурация $4 f^{14} 6 s^{2}$ ) и ионами железа $\mathrm{Fe}^{3+}$ (электронная конфигурация $3 d^{5}$ ). На рис. 3 и 4 представлены результаты магнитных измерений соединения $\mathrm{YbFeTi}_{2} \mathrm{O}_{7}$. 
Таблица 3. Значения асимптотической температуры Нееля $\theta$, константы Кюри-Вейсса $C$ в законе Кюри-Вейсса, расчетные и экспериментальные величины эффективного момента для соединения $\mathrm{YbFeTi}_{2} \mathrm{O}_{7}$

\begin{tabular}{l|c}
\hline \multicolumn{1}{c|}{ Соединение } & $\mathrm{YbFeTi}_{2} \mathrm{O}_{7}$ \\
\hline $\begin{array}{l}\text { Значение температуры } \\
\text { замерзания } T_{f}, \mathrm{~K}\end{array}$ & 4.5 \\
& \\
Значение асимптотической & -127 \\
температуры Нееля $\theta, \mathrm{K}$ & \\
Константа Кюри-Вейсса $C, \mathrm{~K}$ & 0.016 \\
$\mu_{\text {eff саl }}=\left(\mu_{1}^{2}+\mu_{2}^{2}\right)^{1 / 2}$, где & 7.49 \\
$\mu_{i}=g_{i}\left[J_{i}\left(J_{i}+1\right)\right]^{1 / 2}, \mu_{\mathrm{B}}$ & \\
$\mu_{\mathrm{eff} \mathrm{exp}}, \mu_{\mathrm{B}}$ & 7.48
\end{tabular}

Температурный ход обратной магнитной восприимчивости $\chi^{-1}(T)$ для $\mathrm{YbFeTi}_{2} \mathrm{O}_{7}$ в диапазоне температур $2-300 \mathrm{~K}$ при условии охлаждения образца в магнитном поле $H=500$ Ое показан на рис. 3. Анализ температурной зависимости обратной магнитной восприимчивости $\chi^{-1}(T)$ в высокотемпературной области $(T>100 \mathrm{~K})$ показал, что она описывается законом Кюри-Вейсса. В образце в области высоких температур реализуется парамагнитное состояние, характеризуемое отрицательной асимптотической температурой Нееля $\theta=-127 \mathrm{~K}$, что свидетельствует о преимущественно антиферромагнитном взаимодействии в сложной магнитной подсистеме исследуемого образца с $d$ - и $f$-элементами. В табл. 3 приведены экспериментальные значения асимптотической температуры Нееля $\theta$, константы Кюри-Вейсса $C$ в законе Кюри-Вейсса, расчетные и экспериментальные величины эффективного момента для интервала температур, в котором магнитная восприимчивость подчиняется закону Кюри-Вейсса. Расчетное значение эффективного магнитного момента формульной единицы $\mathrm{YbFeTi}_{2} \mathrm{O}_{7}: \mu_{\text {effcal }}=7.48 \mu_{\mathrm{B}}\left(\mu_{\text {effcal }}^{\mathrm{F}^{3+}}=5.91 \mu_{\mathrm{B}}\right.$ и $\mu_{\text {effcal }}^{\mathrm{Yb}^{3+}}=4.59 \mu_{\mathrm{B}}$ ). Константа Кюри-Вейсса $C=0.016 \mathrm{~K}$, что соответствует значению эффективного магнитного момента (молярное значение) $\mu_{\text {effexp }}=4.58 \mu_{\mathrm{B}}$. Определенная из экспериментальных данных величина эффективного магнитного момента сравнима с его расчетным числовым значением.

Магнитные измерения, результаты которых приведены на рис. 4, показывают, что ниже температуры $T_{f}=4.5 \mathrm{~K}$ температурная зависимость магнитного момента $M(T)$ образца $\mathrm{YbFeTi}_{2} \mathrm{O}_{7}$ зависит от предыстории: проводилось ли охлаждение в магнитном поле $H=0.05$ Т или и без него. Таким образом, наблюдаемые зависимости $M(T)$ показывают, что при низких температурах $\left(T<T_{f}=4.5 \mathrm{~K}\right)$ имеется несколько значений магнитного момента в зависимости от условий охлаждения образца. Необходимо отметить, что результаты магнитных измерений в области низких темпера- тур характерны для образцов с магнитным состоянием спинового стекла. По-видимому, атомный беспорядок в распределении железа в кристаллической решетке и случайно изменяющиеся взаимодействия магнитных атомов приводят к образованию при температурах ниже температуры замерзания $T_{f}=4.5 \mathrm{~K}$ спинстекольного магнитного состояния.

Полученные экспериментальные данные, характеризующие магнитные свойства исследуемого соединения, согласуются с результатами экспериментов, выполненных на различных образцах при замещении редкоземельного катиона $R^{3+}$ в системе $R \mathrm{FeTi}_{2} \mathrm{O}_{7}$, где $R=\mathrm{Sm}, \mathrm{Gd}, \mathrm{Dy}$, $\mathrm{Tu}, \mathrm{Tb}, \mathrm{Lu}[1-5]$.

На основании указанных выше особенностей распределения ионов железа в кристаллической решетке, данных магнитных измерений и сравнения со свойствами других соединений ряда $R \mathrm{FeTi}_{2} \mathrm{O}_{7}(R=\mathrm{Sm}, \mathrm{Gd}, \mathrm{Dy}$, $\mathrm{Tu}, \mathrm{Tb}, \mathrm{Lu})$, можно предположить в образце $\mathrm{YbFeTi}_{2} \mathrm{O}_{7}$ при температуре ниже $T_{f}=4.5 \mathrm{~K}$ формирование „замороженного“ пространственного распределения ориентаций спиновых магнитных моментов, обусловленного наличием в системе хаотически расположенных магнитных моментов, конкурирующих взаимодействий и, по-видимому, вызванной ими фрустрации магнитных моментов. Отметим, что оценка уровня фрустраций $\left(F=|\theta| / T_{f}[13]\right)$ показывает высокое его значение, равное 28.2 .

Таким образом, как симметрия кристаллической решетки, так и магнитное состояние в системе $R \mathrm{FeTi}_{2} \mathrm{O}_{7}$ $(R=\mathrm{Yb}, \mathrm{Sm}, \mathrm{Gd}, \mathrm{Dy}, \mathrm{Tu}, \mathrm{Tb}, \mathrm{Lu})$ не зависят от ионного радиуса редкоземельного иона. Формирование магнитных свойств в $\mathrm{YbFeTi}_{2} \mathrm{O}_{7}$ как одного из представителей ряда соединений с общей формулой $R \mathrm{FeTi}_{2} \mathrm{O}_{7}$ обусловлено сложной игрой конкурирующих обменных взаимодействий между магнитными атомными соседями.

\section{4. Заключение}

Методом твердофазной реакции синтезировано новое магнитное соединение $\mathrm{YbFeTi}_{2} \mathrm{O}_{7}$.

Определена кристаллическая структура и доказана неэквивалентность кристаллографических позиций высокоспинового катиона $\mathrm{Fe}^{3+}$ и наличие атомного беспорядка в его распределении в кристаллической решетке $\mathrm{YbFeTi}_{2} \mathrm{O}_{7}$ при комнатной температуре. С учетом относительных заселенностей индивидуальных позиций, кристаллохимическая формула исследуемого соединения имеет вид: $\mathrm{Yb}^{3+} \mathrm{Fe}_{1.00(5)}^{3+} \mathrm{Ti}_{2.00(5)}^{4+} \mathrm{O}_{7}^{2-}$.

Исследование магнитных свойств в интервале температур $2-300 \mathrm{~K}$ показало, что парамагнитное состояние при температурах выше $100 \mathrm{~K}$ описывается законом Кюри-Вейсса и характеризуется отрицательным значением асимптотической температуры Нееля, свидетельствующей о преимущественно антиферромагнитном обменном взаимодействии в спиновой системе соединения $\mathrm{YbFeTi}_{2} \mathrm{O}_{7}$. В области низких температур имеет 
место зависимость намагниченности образца не только от температуры, но и от условий охлаждения (в магнитном поле и без поля). На основании совокупности полученных экспериментальных данных и сравнения со свойствами других ранее изученных соединений ряда $R \mathrm{FeTi}_{2} \mathrm{O}_{7}(R=\mathrm{Sm}, \mathrm{Gd}, \mathrm{Dy}, \mathrm{Tu}, \mathrm{Tb}, \mathrm{Lu})$ предполагаем, что в магнетике $\mathrm{YbFeTi}_{2} \mathrm{O}_{7}$ реализуется магнитное состояние спинового стекла с температурой замерзания $T_{f}=4.5 \mathrm{~K}$.

Экспериментальные данные также свидетельствуют, что варьирование химического давления за счет катионного замещения $R^{3+}$ в системе $R \mathrm{FeTi}_{2} \mathrm{O}_{7}$ не приводит к изменениям симметрии кристаллической структуры и, по-видимому, магнитного состояния.

Полученный новый магнитный материал расширяет класс соединений с высоким уровнем фрустрации и требует проведения дальнейших исследований.

\section{Список литературы}

[1] Г.А. Петраковский, Т.В. Дрокина, А.Л. Шадрина, Д.А. Великанов, О.А. Баюков, М.С. Молокеев, А.В. Карташев, Г.Н. Степанов. ФТТ 53, 1757 (2011).

[2] Г.А. Петраковский, Т.В. Дрокина, Д.А. Великанов, О.А. Баюков, М.С. Молокеев, А.В. Карташев, А.Л. Шадрина, А.А. Мицук. ФТТ 54, 1701 (2012).

[3] T. Drokina, G. Petrakovskii, M. Molokeev, A. Arauzo, J. Bartolome. Phys. Procedia 12, 580 (2015).

[4] T. Drokina, G. Petrakovskii, D. Velikanov, M. Molokeev. Solid State Phenomena 215, 470 (2014).

[5] Т.В. Дрокина, Г.А. Петраковский, М.С. Молокеев, Д.А. Великанов, О.Н. Плетнев, О.А. Баюков. ФТТ 55, 1922 (2013).

[6] I.C. Madsen, R.J. Hill. Adv. X-ray Anal. 35, 39 (1992).

[7] I.C. Madsen, R.J. Hill. J. Appl. Cryst. 27, 385 (1994).

[8] W.I.F. David. Abstract P2.6, NIST Special Publication 846, 210 (1992).

[9] Diffrac-Plus Basic XRD Wizard. Bruker AXS GmbH, Karlsruhe, Germany (2002-2007).

[10] Bruker AXS: TOPAS V4: General profile and structure analysis software for powder diffraction data. - User's Manual, Bruker AXS, Karlsruhe, Germany (2008).

[11] Е.А. Генкина, В.И. Андрианов, Е.Л. Белоконева, Б.В. Милль, Б.А. Максимов, Р.А. Тамазян. Кристаллография 36, 1408 (1991).

[12] К.П. Белов, М.А. Белянчикова, Р.З. Левитин, С.А. Никитин. Редкоземельные ферро- и антиферромагнетики. Наука, М. (1965).

[13] J.A. Mydosh. Spin-Glasses: An Experimental Introduction, Taylor and Francis, N.Y. (1993). 268 p. 